\title{
Fatigue Analysis of Engine Connecting Rod Based on Finite Element Method
}

\author{
Qingguo Luo ${ }^{a}$, Xin Liu \\ Army Academy of Armored Forces, Beijing 100072, China \\ a597139241@qq.com
}

Keywords: engine; connecting rod; finite element; fatigue analysis.

\begin{abstract}
In this paper, ANSYS Workbench 17.0 has been used to model the engine connecting rod and analyze the maximum compression condition of the engine connecting rod. The stress fatigue of engine connecting rod is primarily researched under invariable amplitude load and random load, and the stress, fatigue life and fatigue damage with above cases have been obtained. The presently results provide the basis and reference for the optimization design of the engine connecting rod.
\end{abstract}

\section{Introduction}

Connecting rod is an important part of the engine, it's functions are to connect the piston and the crankshaft, turn the linear motion of the piston into the rotation of the crank movement, and give the force on the piston to the crankshaft [1]. When the engine is working, the motion and the stress of the connecting rod under the effect of the pull, compressive stress and the gas inertial force, are very complicated [2-4]. Therefore, the engine connection rod is prone to have deformation and fatigue fracture. The reliability of the connecting rod has great influence on the life and reliability of engine. The link needs to have sufficient fatigue strength to ensure its working reliability, so it is necessary to analyze the fatigue strength of the connecting rod.

The connecting rod entity model is established based on Pro/E, and then importing it into ANSYS Workbench17.0 to establish the engine connecting rod with finite element analysis model. In addition, the stress fatigue analysis has been carried out under constant amplitude loading and random loading, which is based on statics analysis, the maximum equivalent stress of connecting rod, cloud service life and sensitivity curve have been owned that provide a reference for the optimal design of the connecting rod.

\section{Fatigue Analysis Method}

Under cyclic stress or cyclic strain, the materials and components gradually produce local permanent cumulative damage in one or several places. The process of cracking or sudden rupture after a certain number of cycles is called metal fatigue. The reason that fatigue happens is that the metal is altered by the repeated effects of stress or strain. Although from the microscopic point of view, the generation and development of fatigue cracks are related to the microplastic deformation. However, from a macroscopic perspective, one still divides fatigue failure into high cycle fatigue and low cycle fatigue based on the number of cycles of cycle at which the fatigue failure occurs [5]. Among them, the high cycle fatigue is controlled by the stress amplitude, the cyclic stress level is low, and the elastic deformation occupies the main position. The low cycle fatigue is controlled by strain amplitude and the cyclic stress level is higher, which often takes over the yield of the material to yield the material, leading to the dominance of shaping deformation.

There are different methods of fatigue analysis based on the different forms of fatigue failure. There are three kinds of fatigue analysis methods commonly used in engineering: nominal stress, local stress and damage tolerance [6]. The nominal stress method suitable for high-cycle fatique, local stress strain gauge is suitable for the low cycle fatigue, and damage tolerance method is formed with the development of the application of fracture mechanics and a fatigue analysis method. 


\section{Finite Element Analysis}

\subsection{Three-Dimensional Modeling}

The physical models of engine connecting rod has been modeled in Pro/E, and the connecting rods are simplified during setting up the models, some small chamfer and roundness of connecting rod have been ignored, so it is better to avoid creating singular unit, adding model and the number of nodes in the grid division of connecting rod finite, therefore it is helpful to improve the subsequent resolution accuracy.

\subsection{Finite Element Model}

The connecting rod model is imported from Pro/E to ANSYS Workbench17.0, and the connecting rod material is $42 \mathrm{CrMo}$, the elastic modulus was $200 \mathrm{GPa}$, and the Poisson's ratio was 0.3 , and the density was $7850 \mathrm{~kg} / \mathrm{m} 3$, division of grid, the finite element model for engine connecting rod is shown in figure 1.

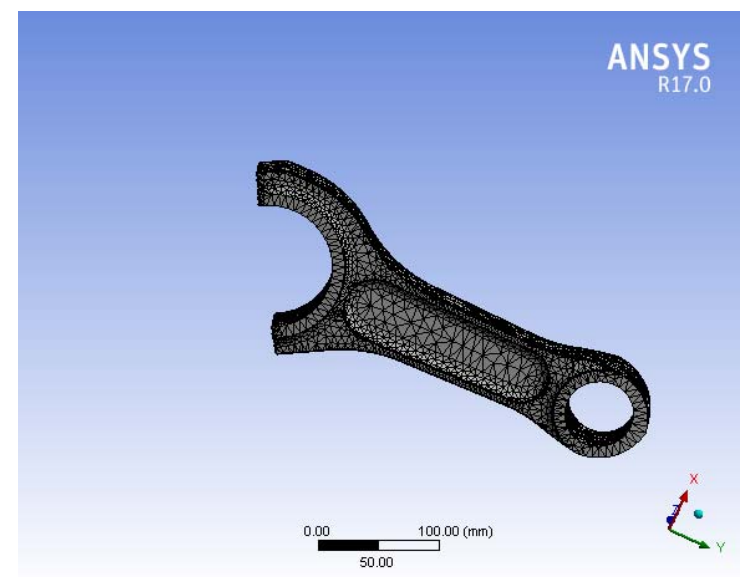

\subsection{Statics Analysis}

FIG.1. Finite element model of engine connecting rod

The simulation is carried out with the maximum compression working condition of the connecting rod to restrict the movement and rotation of connecting rod, complete constraints are imposed on piston pin hole and cylindrical constraints are imposed on both sides of the bolt hole, which make its radial fixed and keep the other two directions to be free [5]. A unit force along the $Y$ axis is applied on the surface of the inner hole of the engine connecting rod. The unit force can be enlarged to perform the fatigue analysis, and the solution can be completed after the setting is completed.

Follow as FIG. 1, you can directly see the equivalent stress distribution of engine connecting rod. The maximum stress appears in the red region of FIG. 1, which is the transition between the large end of the connecting rod and the rod body. When the engine is working, this part of the connecting rod is prone to fatigue damage, resulting in failure.

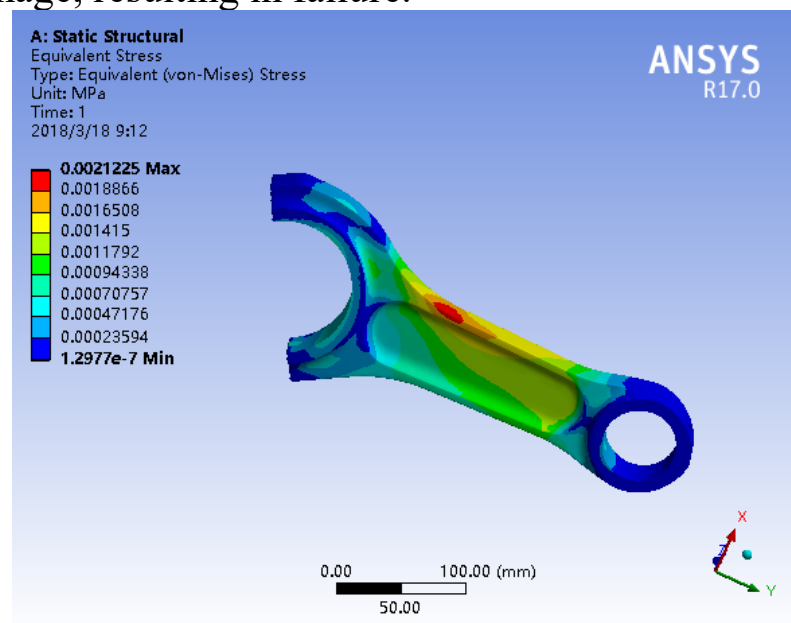

FIG.2. Equivalent stress cloud of engine connecting rod 


\section{Fatigue Analysis}

\subsection{Constant Amplitude Load Fatigue Analysis}

After static analysis, inserting the fatigue analysis tool, amplifying the applied load, setting up safety coefficient, biaxial indication, alternate equivalent stress and fatigue sensitivity, and then calculate the safety factor, biaxial instructions, alternating sensitivity equivalent stress and fatigue of the connecting rod [6].

According to the calculation results, the minimum safety coefficient of the connecting rod is 2.0469 , indicating that the connecting rod is safe. The maximum stress of the connecting rod occurs in the transition place of the head of the connecting rod and the rod body. The stress biaxial direction is the ratio of the minor to the larger principal stress, and the local stress can be determined according to the stress biaxial direction. The maximum ratio is 0.98339 , and the minimum value is -0.99996 , which can be used as an assistant to determine whether the stress state in the key area is similar to the experimental condition. The sensitivity of fatigue curve shows that at about 1.3 times the pressure load, under the action of connecting rod will not occur fatigue damage, the fatigue life of connecting rod is unlimited.

\subsection{Random Load Fatigue Analysis}

Connecting rod's loading is different at different times in the actual work of the engine, therefore, it is not possible to fully reflect the fatigue characteristics of the connecting rod with the constant amplitude load fatigue analysis of the engine connecting rod, and the random load fatigue analysis of the connecting rod should be carried out.

From the results, the minimum safety factor of the connecting rod is 0.70039 , and it needs to be improved and to be strengthened. FIG. 3 shows the life cloud of the engine connecting rod, and the contour line in the cloud diagram represents the cycle times of fatigue failure. The fatigue life of the connecting rod can be analyzed according to the size of the contour line, the maximum fatigue failure of the connecting rod is $3.3693 \mathrm{e} 5$.

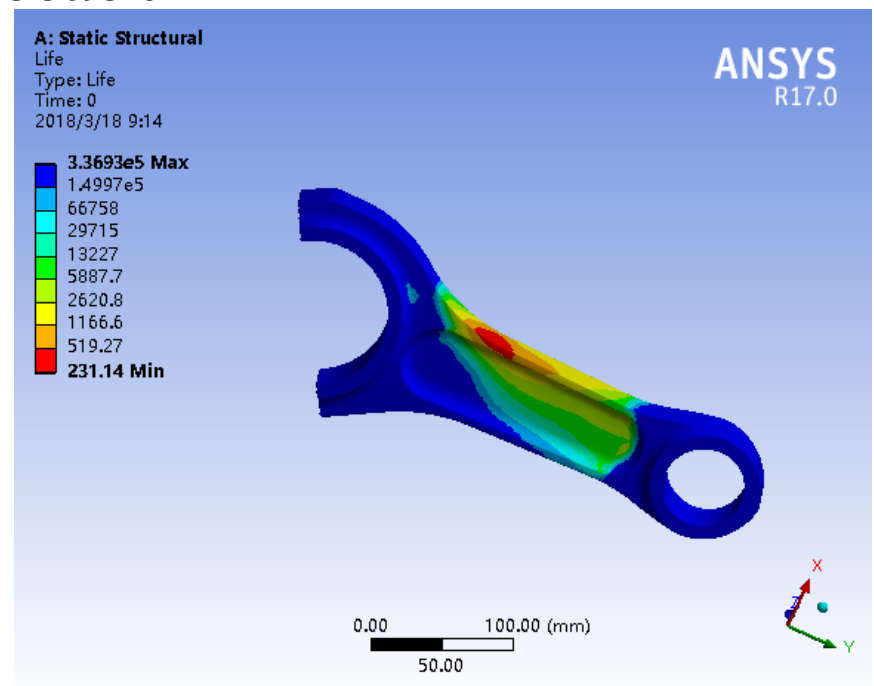

FIG. 3. Life cloud of engine connecting rod

"The rain flow count method" is adopted to improve the irregular load cycle count, the Palmgrn Mine law calculation of fatigue damage accumulation. According to Palmgren-Miner's law, which is, in a given average stress and stress amplitude, the effective life span of each cycle is a few percent of the sum, for a cycle times under a given stress amplitude, the life is exhausted when the number of cycles reaches the failure rate [7].

FIG. 4 shows the cloud map of rain flow matrix. The rain flow matrix can change the alternating stress and average stress into the digital information of the vertical bar. The z-coordinate value represents the size of the count, and the maximum value is 44. FIG. 5 shows the damage matrix cloud map, and the $\mathrm{Z}$ coordinate value of the damage matrix represents the relative damage degree, and the maximum damage degree is 2.18 . The fatigue life and fatigue damage of the connecting rod can be 
calculated by the rain flow matrix and damage matrix of the connecting rod, and the fatigue life of the engine connecting rod under asymmetric load can be predicted [8].

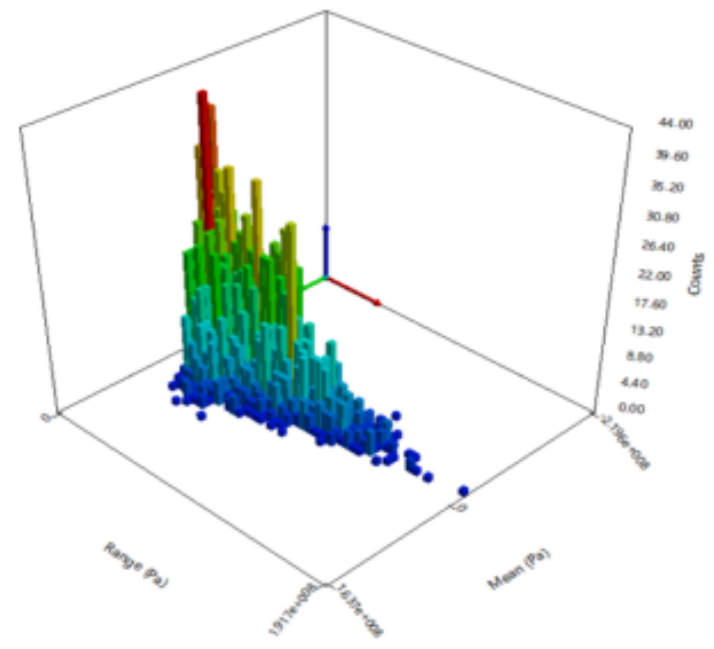

FIG. 4. Rain flow matrix of engine connecting rod

ANSYS

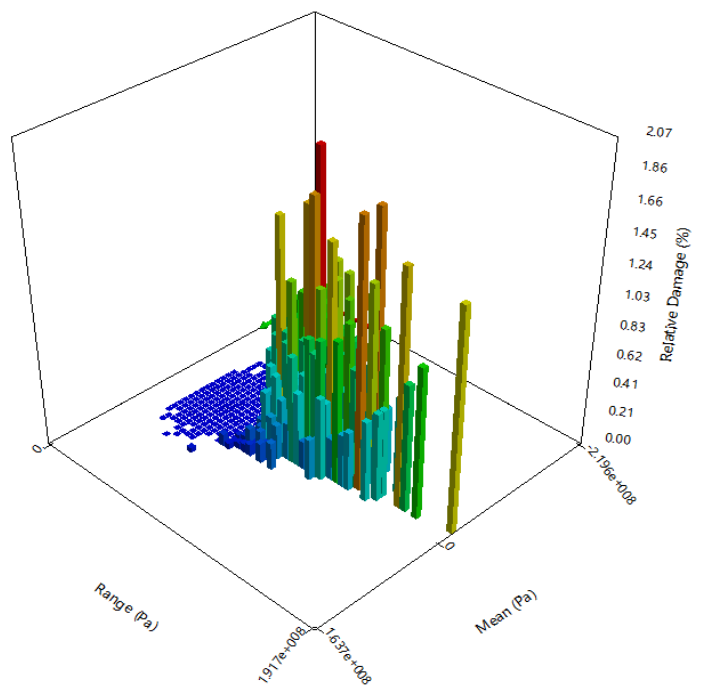

FIG. 5. Fatigue damage matrix of engine connecting rod

\section{Conclusion}

In this paper, ANSYS Workbench17.0 is applied to analyze the statics of engine connecting rod and fatigue analysis. Through simulation, obtained the maximum equivalent stress of engine connecting rod, and further analyzes the safety coefficient, fatigue life and fatigue damage of the connecting rod, the presently results provide a reference for the optimal design of the connecting rod. The application of ANSYS to carry out simulation calculation not only reduces the input of manpower and material resources, but also saves the development cost and shorten the development cycle.

\section{References}

[1]. Yang Z B, Luo P F, Liu K F. Optimal design of engine connecting rod based on FEM [J]. Journal of Henan Polytechnic University, 2013.

[2]. Wang Y. Study on Fatigue Strength Analysis of Engine Linkage based on FEA [J]. Journal of Mechanical Transmission, 2010. 
[3]. Liu Y, Hu Q, Zhang R, et al. Finite Element Analysis of Connecting Rod of Mud Pump Based on ANSYS[J]. Agricultural Equipment \& Vehicle Engineering, 2013.

[4]. Wang X Y, Yuan S C, Liu J. Fatigue Life Estimation Study of Connecting Rod Based on AWE [J]. Coal Mine Machinery, 2010.

[5]. Wang Y W, Luo J W, Jun Y E, et al. FEA based fatigue analysis and Its application[J]. Machinery Design \& Manufacture, 2008.

[6]. Guo J. Finite-element Modal Analysis of Engine Crankshaft Based on ANSYS Workbench [J]. Natural Science Journal of Harbin Normal University, 2017.

[7]. Fatigue Strength Analysis of Diesel Engine Connecting Rod Based on Finite Element Analysis [J]. Internal Combustion Engine \& Parts, 2011.

[8]. Chen N Z, Wang G, Soares C G. Palm Gren - Miner's rule and fracture mechanics-based inspection planning [J]. Engineering Fracture Mechanics, 2011, 78(18):3166-3182.

[9]. Zhu R F, Zhao Q F, Wang H. Fatigue strength analysis of engine connecting rod based on ANSYS Workbench[J]. Journal of Heilongjiang Institute of Technology, 2014. 